\title{
Increased serum cholesterol and long-chain fatty acid levels are associated with the efficacy of nivolumab in patients with non-small cell lung cancer
}

\author{
Masato Karayama ${ }^{1,2}$ (D) Naoki Inui ${ }^{3} \cdot$ Yusuke Inoue $^{2} \cdot$ Katsuhiro Yoshimura $^{2} \cdot$ Kazutaka Mori $^{4} \cdot$ Hironao Hozumi $^{2} \cdot$ \\ Yuzo Suzuki ${ }^{2} \cdot$ Kazuki Furuhashi $^{2} \cdot$ Tomoyuki Fujisawa $^{2} \cdot$ Noriyuki Enomoto $^{2} \cdot$ Yutaro Nakamura $^{2} \cdot$ Kazuhiro Asada $^{5}$. \\ Tomohiro Uto $^{6}$. Masato Fujii ${ }^{7}$. Takashi Matsui ${ }^{8}$. Shun Matsuura ${ }^{9}$. Dai Hashimoto ${ }^{10}$ - Mikio Toyoshima ${ }^{11}$. \\ Hideki Kusagaya ${ }^{12} \cdot$ Hiroyuki Matsuda $^{13} \cdot$ Nao Inami $^{4} \cdot$ Yusuke Kaida $^{14} \cdot$ Mitsuru Niwa $^{15} \cdot$ Yasuhiro Ito $^{16}$. \\ Takafumi Suda ${ }^{2}$
}

Received: 10 March 2021 / Accepted: 1 June 2021 / Published online: 5 June 2021

(c) The Author(s) 2021

\begin{abstract}
Background Lipids have immunomodulatory functions and the potential to affect cancer immunity.

Methods The associations of pretreatment serum cholesterol and long-chain fatty acids with the objective response rate (ORR), progression-free survival (PFS), and overall survival (OS) were evaluated in 148 patients with non-small cell lung cancer who received nivolumab.

Results When each lipid was separately evaluated, increased low-density lipoprotein $($ LDL $)$-cholesterol $(P<0.001)$, high-density lipoprotein (HDL)-cholesterol $(P=0.014)$, total cholesterol $(P=0.007)$, lauric acid $(P=0.015)$, myristic acid $(P=0.022)$, myristoleic acid $(P=0.035)$, stearic acid $(P=0.028)$, linoleic acid $(P=0.005)$, arachidic acid $(P=0.027)$, eicosadienoic acid $(P=0.017)$, dihomo- $\gamma$-linolenic acid $(P=0.036)$, and behenic acid levels $(P=0.032)$ were associated with longer PFS independent of programmed death ligand 1 (PD-L1) expression. Meanwhile, increased LDL-cholesterol $(P<0.001)$, HDL-cholesterol $(P=0.009)$, total cholesterol $(P=0.036)$, linoleic acid $(P=0.014)$, and lignoceric acid levels $(P=0.028)$ were associated with longer OS independent of PD-L1 expression. When multiple lipids were evaluated simultaneously, LDL-cholesterol $(P=0.003)$, HDL-cholesterol $(P=0.036)$, and lauric acid $(P=0.036)$ were independently predictive of PFS, and LDL-cholesterol $(P=0.008)$ and HDL-cholesterol $(P=0.031)$ were predictive of OS. ORR was not associated with any serum lipid.

Conclusions Based on the association of prolonged survival in patients with increased serum cholesterol and long-chain fatty acid levels, serum lipid levels may be useful for predicting the efficacy of immune checkpoint inhibitor therapy.
\end{abstract}

Keywords Anti-programmed death-1 therapy $\cdot$ Immunometabolism $\cdot$ Immune checkpoint inhibitor $\cdot$ Lipid $\cdot$ Metabolism

\section{Introduction}

Immune checkpoint inhibitors (ICIs) are increasingly used as new standard treatments for cancers, including nonsmall cell lung cancer (NSCLC) $[1,2]$. However, only some patients benefit from ICI therapy, and the benefits are not sustained in most responders. To address unmet needs, novel biomarkers and/or combination therapies that can enhance

Masato Karayama

karayama@hama-med.ac.jp

Extended author information available on the last page of the article the efficacy of ICIs have been intensively investigated [3, 4]. A better understanding of factors associated with the therapeutic benefits of ICIs could provide improved precision medicine and new insights into the mechanisms of response and resistance to ICIs.

Unlike cytotoxic chemotherapy with direct anti-tumor effects, ICIs induce anti-tumor responses via immune cells. Therefore, to understand the therapeutic effects of ICIs, assessments of host-tumor interactions are essential in addition to tumor characteristics. It has become increasingly evident that the functions of immune cells are strongly associated with metabolism. Specifically, metabolites have essential roles in controlling the function, differentiation, or 
longevity of immune cells, thereby affecting cancer immunity [5-11]. For examples, tryptophan and its metabolites are known to affect cancer immunity [12, 13]. Decreased tryptophan levels induce anergy in effector T cells, and increased levels of the tryptophan metabolite kynurenine result in regulatory $\mathrm{T}$ cell activation and attenuate anti-tumor immunity [12]. Previously, we found that decreased levels of 3-hydroxyanthranilic acid, a downstream metabolite of the tryptophan-kynurenine pathway, are associated with longer progression-free survival (PFS) in nivolumab-treated patients with NSCLC [14]. The evaluation of immunometabolism will provide important information for ICI therapy.

Lipids, which serve as energy resources and components of the cell membrane, have immunomodulatory potential. Lipids are necessary for immune cell activation, including effector T cells, natural killer T cells, macrophages, and dendritic cells [5-10]. Decreased cholesterol levels inhibited the proliferation and activation of $\mathrm{T}$ cells [15]. Inversely, increased cholesterol content fostered Th1 differentiation [16]. Lauric acid, a long-chain fatty acid, induced the polarization of naïve T cells toward Th1 and Th17 cells [17, 18], and it activated bone marrow-derived dendritic cells and increased $\mathrm{T}$ cell activation capacity [19]. Cancer cells require lipids for their proliferation and progression; therefore, these cells aggressively compete with immune cells for lipid uptake. Consequently, cancer cells deprive immune cells of these essential lipids and inhibit their activation [7, 20-24].

However, little is known about the roles of serum lipids in ICI efficacy. The influence of fatty acids on immunity differs by type. It is well known that $\omega-3$ polyunsaturated fatty acids (PUFAs) promote anti-inflammatory responses, whereas $\omega-6$
PUFAs and saturated fatty acids promote pro-inflammatory responses [19, 25-27]. Given the diverse immunoregulatory functions of lipids, we hypothesized that different lipids have different effects on the efficacy of ICI therapy. The current study assessed the pretreatment serum levels of multiple lipids and evaluated their associations with the efficacy of nivolumab in patients with previously treated NSCLC. In addition, we also evaluated pretreatment sera from patients with NSCLC who received cytotoxic chemotherapy.

\section{Patients and methods}

\section{Study design}

This was a post hoc analysis of a prospective, multicenter, observational study conducted in 14 hospitals in Japan between July 1, 2016, and December 11, 2018 [28]. Each patient provided written informed consent. Additionally, a retrospective cohort of patients who received cytotoxic chemotherapy (chemotherapy cohort) treated at Hamamatsu University Hospital between January 1, 2009, and December 31,2019 , was evaluated. The study followed the ethical standards of the Declaration of Helsinki. The study protocol was approved by the Institutional Review Board of Hamamatsu University School of Medicine (No. 19-083). The study was registered with the University Hospital Medical Information Network Clinical Trial Registry $(000,039,188)$. This study followed the Strengthening the Reporting of Observational Studies in Epidemiology reporting guideline [29].

\begin{tabular}{|c|c|}
\hline & $N=148$ \\
\hline Age, years & $69(63-74)$ \\
\hline Sex, men & $122(82.4)$ \\
\hline Smoking status, ever-smoker & $131(88.5)$ \\
\hline Body mass index, $\mathrm{kg} / \mathrm{m}^{2}$ & $21.2(19.0-23.0)$ \\
\hline Use of statins & $20(13.5)$ \\
\hline ECOG-PS, $0 / 1 / \geq 2$ & $78(52.7) / 62(41.9) / 8(5.4)$ \\
\hline Stage, IIIb/IV/recurrence & $33(22.3) / 101(68.2) / 14(9.5)$ \\
\hline Pathology, adeno/squamous/others & $81(54.7) / 55(37.1) / 12(8.1)$ \\
\hline PD-L1 expression: TPS, $<1 \% / 1-49 \% / \geq 50 \% /$ unknown & $74(50.0) / 49(33.1) / 21(14.2) / 4(2.7)$ \\
\hline EGFR mutation, positive/wild-type/unknown & $8(5.4) / 110(74.3) / 30(20.3)$ \\
\hline$A L K$ fusion gene, positive/wild-type/unknown & $1(0.7) / 110(74.3) / 37(25.0)$ \\
\hline Treatment line, $2 \mathrm{nd} / \geq 3 \mathrm{rd}$ & $82(55.4) / 66(44.6)$ \\
\hline
\end{tabular}

Data are expressed as the median (interquartile range) or number (\%)

$A L K$ anaplastic lymphoma kinase; ECOG-PS Eastern Cooperative Oncology Group performance status; $E G F R$ epidermal growth factor receptor; $P D-L 1$ programmed death ligand-1; TPS tumor proportion score 


\section{Patients}

The study inclusion criteria have been described in a previous publication [28]. Briefly, patients with an Eastern Cooperative Oncology Group performance status (ECOG-PS) of 0-2 who were scheduled to receive nivolumab monotherapy for previously treated advanced NSCLC were included. Patients lacking pretreatment serum samples were excluded. Response was assessed every four cycles by local investigators using Response Evaluation Criteria in Solid Tumors (RECIST) version 1.1.

The chemotherapy cohort included patients with advanced NSCLC who received first-line platinum-based chemotherapies. Patients who received non-platinum therapy or had histories of previous chemotherapy or ICI therapy were excluded. The clinical data were retrospectively evaluated via medical record review.

\section{Lipid measurements}

Low-density lipoprotein (LDL)-cholesterol and high-density lipoprotein (HDL)-cholesterol levels were evaluated by the direct method $\left(\right.$ Choletest ${ }^{\circledR}$ LDL and Choletest ${ }^{\circledR} \mathrm{N}$ HDL, respectively; SEKISUI Medical, Tokyo, Japan). Total cholesterol content was evaluated using an enzymatic method (Choletest ${ }^{\circledR} \mathrm{CHO}$, SEKISUI Medical). Meanwhile, 24 long-chain fatty acids were evaluated via gas chromatography (GC-2010, Shimadzu, Kyoto, Japan): lauric acid, myristic acid, myristoleic acid, palmitic acid, palmitoleic acid, stearic acid, oleic acid, linoleic acid, $\gamma$-linolenic acid, linolenic acid, arachidic acid, eicosenoic acid, eicosadienoic acid, eicosatrienoic acid, dihomo- $\gamma$-linolenic acid, arachidonic acid, eicosapentaenoic acid, behenic acid, erucic acid, docosatetraenoic acid, docosapentaenoic acid, lignoceric acid, docosahexaenoic acid, and nervonic acid. Erucic acid was not included because its levels were below the limit of
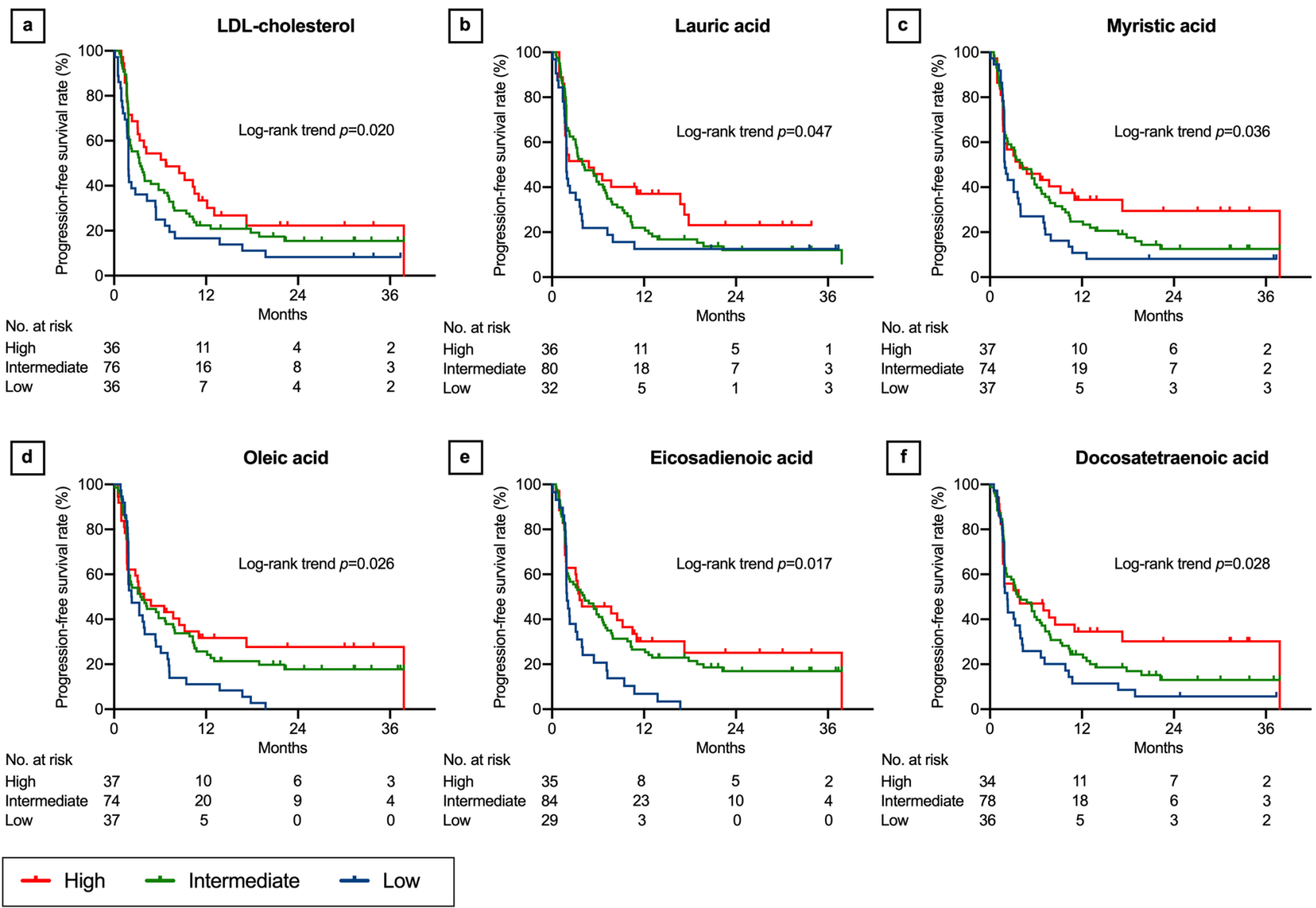

Fig. 1 Progression-free survival after nivolumab therapy according to serum lipid levels: part 1. Low-density lipoprotein (LDL) cholesterol (a), lauric acid (b), myristic acid (c), oleic acid (d), eicosadienoic acid (e), and docosatetraenoic acid (f). Three concentration catego- ries were created for each lipid: low (less than the interquartile range [IQR], blue line), intermediate (within the IQR, green line), and high (higher than the IQR, red line) 


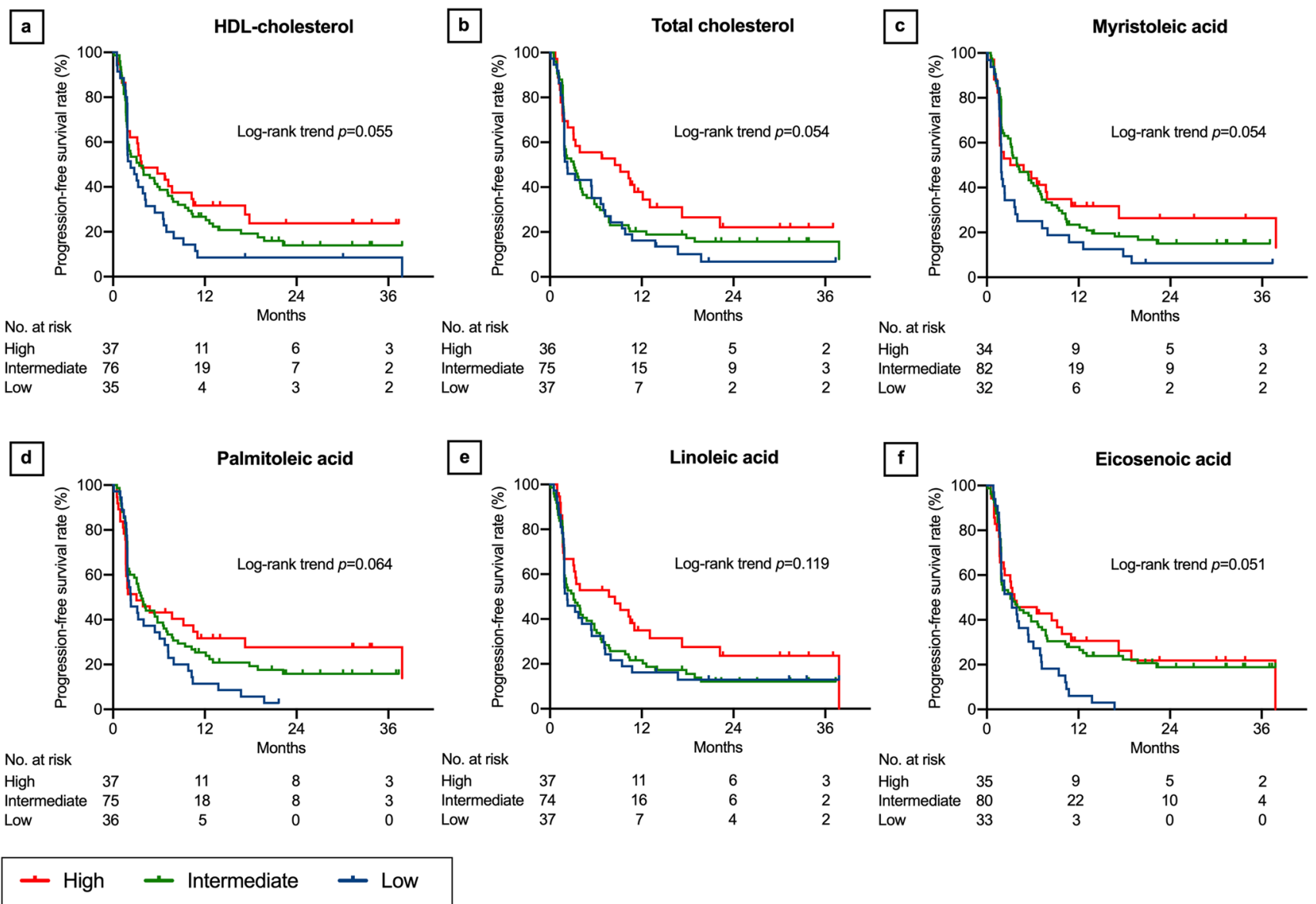

Fig. 2 Progression-free survival after nivolumab therapy according to serum lipid levels: part 2. High-density lipoprotein (HDL) cholesterol (a), total cholesterol (b), myristoleic acid (c), palmitoleic acid (d), linoleic acid (e), and eicosenoic acid (f). Three concentration catego-

detection in most patients. All measurements were taken at a laboratory (SRL, Inc., Tokyo, Japan) certified by the College of American Pathologist and International Organization for Standardization 15,189 .

\section{Evaluation of clinical features and outcomes}

Clinical factors, including age, sex, smoking status, body mass index (BMI), pathology, clinical stage, ECOG-PS, and line of treatment, were recorded. Tumor-programmed death ligand-1 (PD-L1) protein expression was evaluated immunohistochemically using the tumor proportion score (TPS. E1L3N antibody (Cell Signaling Technology) was used for PD-L1 immunohistochemistry until Japanese approval of the $22 \mathrm{C} 3$ pharmDX assay (Agilent). The objective response rate (ORR, sum of the partial and complete response rates was evaluated using RECIST version 1.1.), PFS and overall survival (OS) were evaluated from the time of treatment initiation. ries were created for each lipid: low (less than the interquartile range [IQR], blue line), intermediate (within the IQR, green line), and high (higher than the IQR, red line)

\section{Statistical analyses}

Student's $t$ test and Fisher's exact test were used for continuous and categorical variables, respectively. Pearson's correlation analysis was used to assess the correlations between lipid levels and clinical factors. Kaplan-Meier analyses were used for PFS and OS. In these analyses, lipid levels were divided into three groups by concentration as follows: low (below the interquartile range [IQR]), intermediate (within the IQR), and high (exceeding the IQR). The log-rank trend test was used to examine the associations of PFS or OS with stepwise increases in lipid levels. Logistic regression analysis was used to evaluate predictive factors for ORR, and the Cox proportional hazard analysis was used to assess predictors of PFS and OS. Variables significant at $P<0.100$ in univariate analyses were employed for multivariate analyses. Additionally, sex and ECOG-PS were associated with lipid levels; therefore, they were included in the multivariate analyses. Two types of multivariate analysis were performed using different combinations of variables. First, to evaluate 

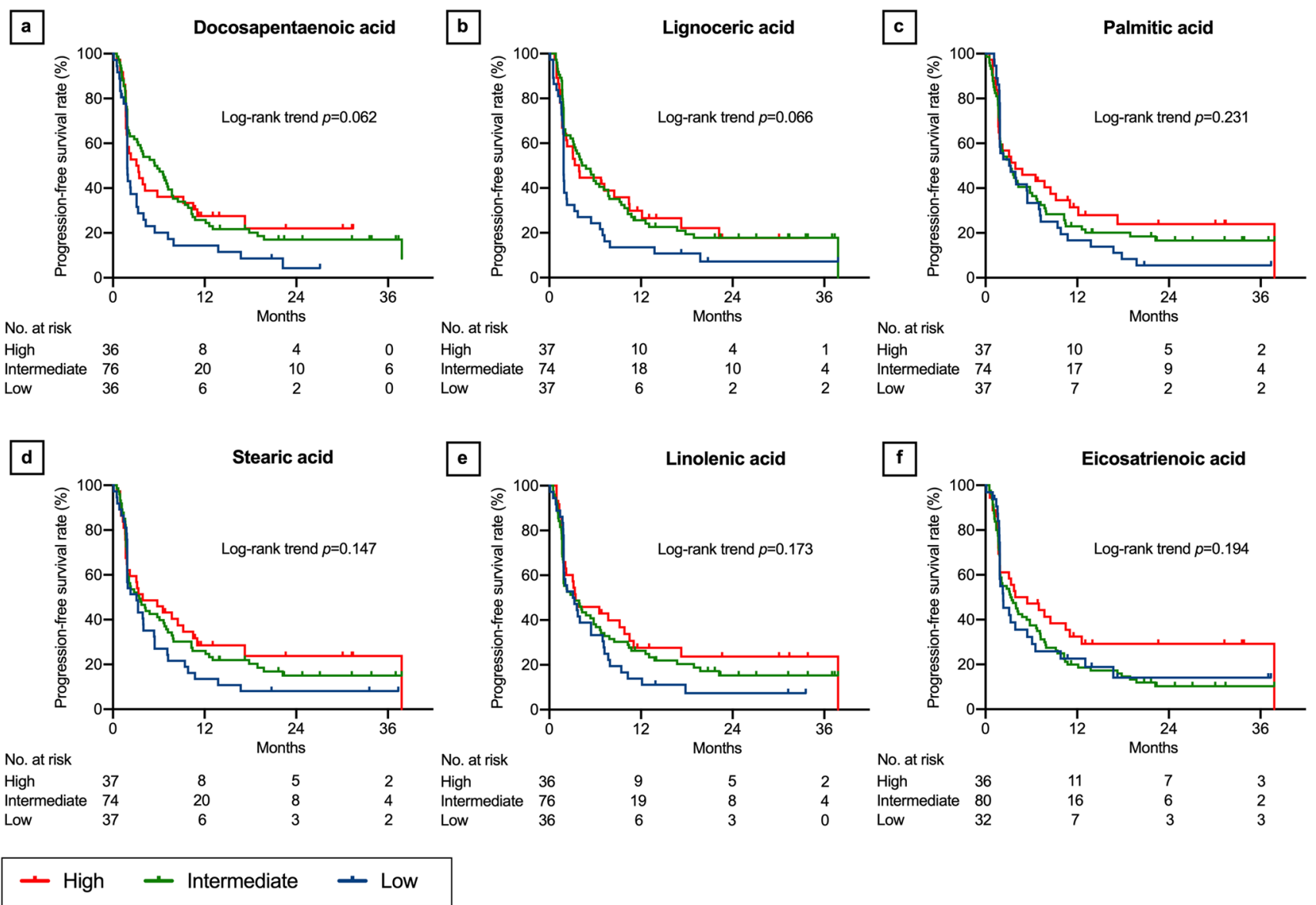

Fig. 3 Progression-free survival after nivolumab therapy according to serum lipid levels: part 3. Docosapentaenoic acid (a), lignoceric acid (b), palmitic acid (c), stearic acid (d), linolenic acid (e), and eicosatrienoic acid (f). Three concentration categories were created for each

predictive utility of a single lipid, each individual lipid was analyzed with adjustment for sex, ECOG-PS, and variables significant at $P<0.100$ in univariate analysis (single-lipid analysis). Second, to identify major contributing factors among the lipids, multiple lipids significant at $P<0.100$ in univariate analysis were analyzed together with adjustment for sex, ECOG-PS, and variables significant at $P<0.100$ in univariate analysis (multiple-lipid analysis). When variables had strong correlations with each other (Pearson's correlation coefficient $>0.7$ ), only one was selected for the multiple-lipid analysis to avoid multicollinearity. Candidate combinations for multiple-lipid analysis were created by grouping lipids without strong correlations with each other. Among the candidate combinations, the best model based on the Akaike information criterion was selected as a representative combination of lipids (Supplementary Methods). $P<0.05$ (two-sided) denoted significance. All values were analyzed using JMP v13.2.0 (SAS Institute Japan, Tokyo, Japan), excluding log-rank trend test data, which were lipid: low (less than the interquartile range [IQR], blue line), intermediate (within the IQR, green line), and high (higher than the IQR, red line)

analyzed using PRISM Version 7.0d (GraphPad Software, CA, USA).

\section{Results}

\section{Patient characteristics}

Among 200 patients who were enrolled in the original prospective study, 52 patients who did not have sufficient serum samples for lipid measurements were excluded. Therefore, 148 patients with assessable pretreatment serum samples were included in this post hoc analysis. Patient characteristics are presented in Table 1. Most patients were men $(82.4 \%)$, and most had a history of smoking $(88.5 \%)$ and ECOG-PS of 0-1 (94.6\%). Ninety-three patients $(62.8 \%)$ had non-squamous cell carcinoma. Tumor PD-L1 expression was assessed in 144 patients (97.3\%), and the TPS was $1 \%-49 \%$ in 49 patients $(33.1 \%)$ and $\geq 50 \%$ in 21 patients (14.2\%). One hundred and thirty-eight patients (93.2\%) 


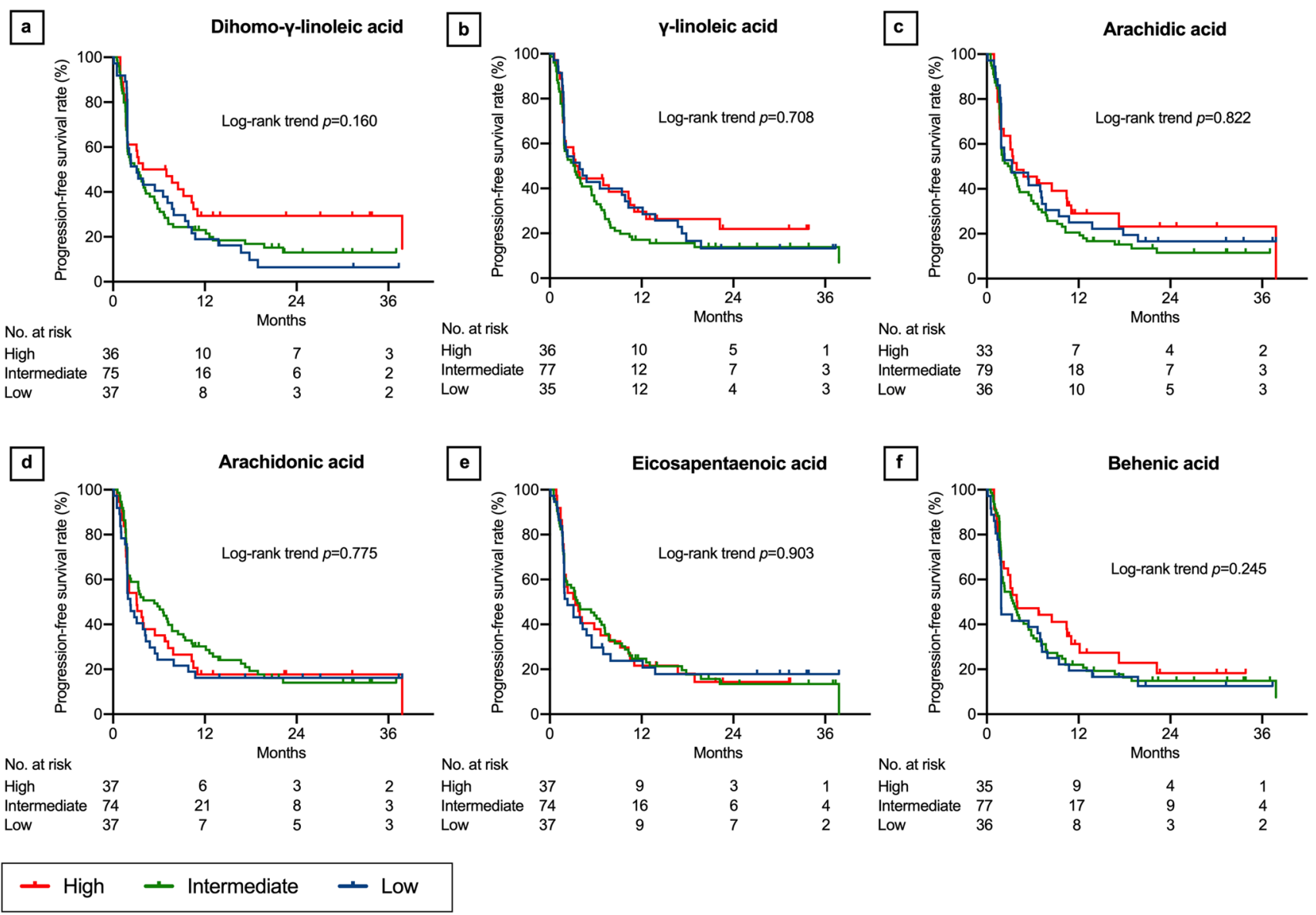

Fig. 4 Progression-free survival after nivolumab therapy according to serum lipid levels: part 4. Dihomo- $\gamma$-linoleic acid (a), $\gamma$-linoleic acid (b), arachidic acid (c), arachidonic acid (d), eicosapentaenoic acid (e), and behenic acid (f). Three concentration categories were created

received platinum-based therapies before nivolumab, and $82(55.4 \%)$ received nivolumab as a second-line therapy. Overall ORR was $22.3 \%$, and median PFS and OS were 3.3 $(95 \%$ confidence interval $[\mathrm{CI}]=2.1-5.4)$ and 14.8 months $(95 \% \mathrm{CI}=12.8-19.7)$, respectively.

\section{Associations of lipid levels with patient demographics}

Women had significantly higher LDL-cholesterol $(P=0.005)$, HDL-cholesterol $(P=0.006)$, total cholesterol $(P=0.001)$, myristic acid $(P=0.042)$, palmitic acid $(P=0.008)$, stearic acid $(P=0.001)$, oleic acid $(P=0.014)$, linoleic acid $(P<0.001), \gamma$-linolenic acid $(P=0.007)$, linolenic acid $(P<0.001)$, arachidic acid $(P=0.017)$, eicosadienoic acid $(P=0.016)$, eicosapentaenoic acid $(P=0.034)$, behenic acid $(P=0.014)$, docosapentaenoic acid $(P$ $<0.001)$, and docosahexaenoic acid levels $(P<0.001$, Supplementary Table 1). Patients with ECOG-PS of 0-1 had significantly higher LDL-cholesterol $(P=0.032)$, myristic for each lipid: low (less than the interquartile range [IQR], blue line), intermediate (within the IQR, green line), and high (higher than the IQR, red line). The Kaplan-Meier curves of docosahexaenoic acid and nervonic acid are presented in Supplementary Fig. 1

acid $(P=0.021)$, myristoleic acid $(P=0.049)$, stearic acid $(P=0.038)$, linolenic acid $(P=0.042)$, dihomo- $\gamma$-linolenic acid $(P=0.013)$, behenic acid $(P=0.025)$, and lignoceric acid levels $(P=0.005)$ and significantly lower nervonic acid levels $(P=0.009)$ than those with ECOG-PS $\geq 2$ (Supplementary Table 2). Lipid levels were not correlated with age or BMI (Supplementary Table 3). Strong correlations were noted between total cholesterol and LDL-cholesterol $(r=0.86)$, myristic acid and myristoleic acid $(r=0.88)$, palmitic acid and stearic acid $(r=0.92)$, palmitic acid and oleic acid $(r=0.94)$, oleic acid and stearic acid $(r=0.86)$, and lignoceric acid and behenic acid $(r=0.94)$. Correlations among other lipids are presented in Supplementary Table 3.

\section{Association of lipid levels with PFS}

In the log-rank trend analyses of the Kaplan-Meier curves, PFS increased with increasing LDL-cholesterol $(P=0.020$, Fig. 1a), lauric acid $(P=0.047$, Fig. $1 b)$, myristic acid $(P=0.036$, Fig. $1 \mathrm{c})$, oleic acid $(P=0.026$, Fig. $1 \mathrm{~d})$, 
Table 2 Multivariate Cox proportional hazard analyses of progression-free survival

\begin{tabular}{|c|c|c|c|c|}
\hline \multirow[b]{2}{*}{ Variables } & \multicolumn{2}{|c|}{ Single-lipid analysis } & \multicolumn{2}{|c|}{ Multiple-lipid analysis } \\
\hline & Hazard ratio & $P$ & Hazard ratio & $P$ \\
\hline LDL-cholesterol $^{\mathrm{a}}$ & $0.87(0.82-0.93)$ & $<0.001$ & $0.86(0.78-0.94)$ & 0.001 \\
\hline HDL-cholesterol $^{\mathrm{a}}$ & $0.81(0.68-0.95)$ & 0.014 & $0.83(0.70-0.98)$ & 0.027 \\
\hline Total cholesterol $^{\mathrm{a}}$ & $0.94(0.90-0.98)$ & 0.007 & & \\
\hline Lauric acid ${ }^{\mathrm{b}}$ & $0.80(0.61-0.96)$ & 0.015 & $0.80(0.58-0.99)$ & 0.040 \\
\hline Myristic acid ${ }^{b}$ & $0.89(0.81-0.98)$ & 0.022 & & \\
\hline Myristoleic acid & $0.86(0.76-0.99)$ & 0.035 & $0.96(0.80-1.13)$ & 0.653 \\
\hline Palmitic acid ${ }^{\mathrm{d}}$ & $0.93(0.85-1.01)$ & 0.100 & & \\
\hline Palmitoleic acid ${ }^{\mathrm{b}}$ & $0.96(0.91-1.02)$ & 0.209 & & \\
\hline Stearic acid ${ }^{\mathrm{d}}$ & $0.70(0.51-0.96)$ & 0.028 & $1.83(0.95-3.46)$ & 0.069 \\
\hline Oleic acid ${ }^{\mathrm{d}}$ & $0.94(0.87-1.02)$ & 0.150 & & \\
\hline Linoleic acid ${ }^{\mathrm{d}}$ & $0.88(0.81-0.96)$ & 0.005 & & \\
\hline$\gamma$-linolenic acid ${ }^{c}$ & $0.98(0.95-1.01)$ & 0.141 & & \\
\hline Linolenic acid ${ }^{\mathrm{c}}$ & $0.99(0.97-1.00)$ & 0.061 & & \\
\hline Arachidic acid ${ }^{c}$ & $0.91(0.84-0.99)$ & 0.027 & & \\
\hline Eicosenoic acid ${ }^{c}$ & $0.94(0.87-1.02)$ & 0.152 & $0.94(0.84-1.06)$ & 0.324 \\
\hline Eicosadienoic acid ${ }^{c}$ & $0.90(0.83-0.98)$ & 0.017 & & \\
\hline Eicosatrienoic acid ${ }^{\mathrm{c}}$ & $0.95(0.87-1.04)$ & 0.245 & & \\
\hline Dihomo- $\gamma$-linolenic acid ${ }^{c}$ & $0.98(0.97-0.99)$ & 0.036 & $1.00(0.98-1.02)$ & 0.818 \\
\hline Arachidonic acid ${ }^{\mathrm{b}}$ & $0.97(0.94-1.01)$ & 0.150 & & \\
\hline Eicosapentaenoic acid ${ }^{b}$ & $0.99(0.93-1.06)$ & 0.867 & & \\
\hline Behenic acid ${ }^{c}$ & $0.96(0.92-0.99)$ & 0.032 & & \\
\hline Docosatetraenoic acid & $0.93(0.84-1.02)$ & 0.129 & & \\
\hline Docosapentaenoic acid & $0.98(0.95-1.01)$ & 0.121 & & \\
\hline Lignoceric acid ${ }^{c}$ & $0.96(0.93-1.00)$ & 0.072 & & \\
\hline Docosahexaenoic acid ${ }^{b}$ & $0.96(0.91-1.00)$ & 0.080 & & \\
\hline Nervonic acid $^{\mathrm{b}}$ & $0.89(0.74-1.07)$ & 0.227 & & \\
\hline
\end{tabular}

The hazard ratio was adjusted for sex, smoking status, Eastern Cooperative Oncology Group performance status, programmed death ligand-1 expression, and treatment line (2nd vs. $\geq 3 \mathrm{rd}$ ). Total cholesterol, myristoleic acid, and linoleic acid were excluded from the multiple-lipid analysis because of their strong correlations with other lipids, and the results of the other combinations of the excluded lipids are presented in Supplementary Table 5

${ }^{a}$ per $10 \mathrm{mg} / \mathrm{dL}$ increase

${ }^{\mathrm{b}}$ per $10 \mathrm{mg} / \mathrm{dL}$ increase

${ }^{c}$ per $1 \mathrm{mg} / \mathrm{dL}$ increase

d per $100 \mathrm{mg} / \mathrm{dL}$ increase eicosadienoic acid ( $P=0.017$, Fig. 1e), and docosatetraenoic acid levels $(P=0.028$, Fig. 1f). Although not statistically significant, PFS tended to increase with increasing HDLcholesterol ( $P=0.055$, Fig. $2 \mathrm{a})$, total cholesterol $(P=0.054$, Fig. 2b), myristoleic acid $(P=0.054$, Fig. 2c), palmitoleic acid $(P=0.064$, Fig. 2 d), linoleic acid $(P=0.119$, Fig. 2e), eicosenoic acid $(P=0.051$, Fig. 2f $)$, docosapentaenoic acid $(P=0.062$, Fig. 3a), lignoceric acid $(P=0.066$, Fig. 3b), palmitic acid $(P=0.231$, Fig. $3 c)$, stearic acid $(P=0.147$, Fig. 3d), linolenic acid $(P=0.173$, Fig. 3e), eicosatrienoic acid $(P=0.194$, Fig. 3f), and dihomo- $\gamma$-linoleic acid levels $(P=0.160$, Fig. 4a). There was no association of PFS with $\gamma$-linoleic acid $(P=0.708$, Fig. $4 b)$, arachidic acid
$(P=0.822$, Fig. 4c), arachidonic acid $(P=0.775$, Fig. $4 d)$, eicosapentaenoic acid $(P=0.903$, Fig. 4e), behenic acid $(P=0.245$, Fig. 4f), docosahexaenoic acid $(P=0.481$, Supplementary Fig. 1a), and nervonic acid level $(P=0.500$, Supplementary Fig. 1b).

In univariate Cox proportional hazard analyses, increased LDL-cholesterol $(P=0.004)$, HDL-cholesterol $(P=0.019)$, total cholesterol $(P=0.042)$, lauric acid $(P=0.020)$, myristic acid $(P=0.026)$, myristoleic acid $(P=0.038)$, linoleic acid $(P=0.048)$, and eicosadienoic acid levels $(P=0.040)$ were predictive of longer PFS, similarly as smoking status, ECOG-PS, PD-L1 expression, and line of treatment (Supplementary Table 4). When each single lipid was analyzed 
a

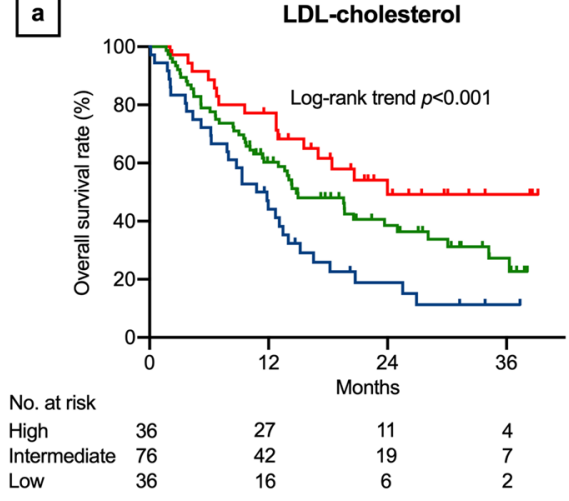

b

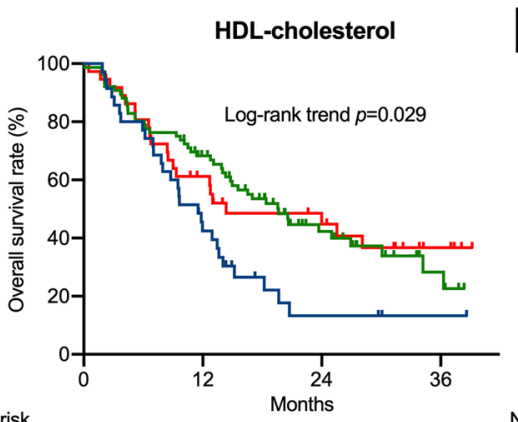

No. at risk

High $\quad 37$

High $\quad 37$

$\begin{array}{ll}\text { Low } & 35\end{array}$
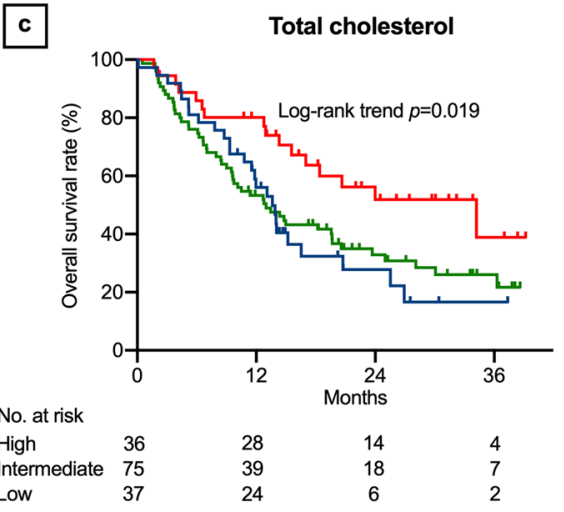
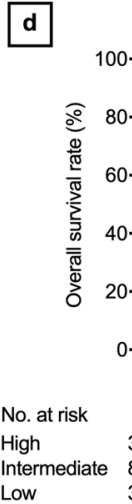

Lauric acid

Log-rank trend $p=0.032$

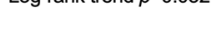

(3)
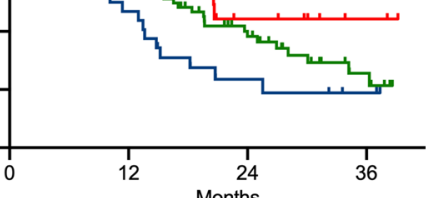

$\begin{array}{lllcl}\text { High } & 36 & 19 & 8 & 3 \\ \text { Intermediate } & 80 & 50 & 22 & 7 \\ \text { Low } & 32 & 16 & 6 & 3\end{array}$ e

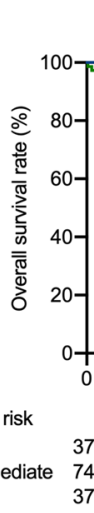

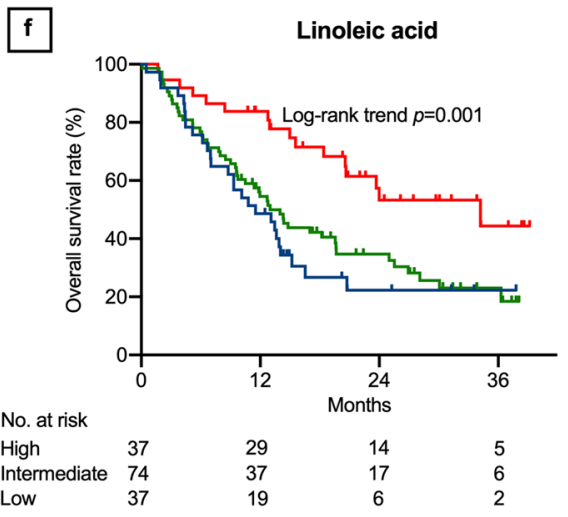

- High — Intermediate — Low

Fig. 5 Overall survival after nivolumab therapy according to serum lipid levels: part 1. Low-density lipoprotein (LDL) cholesterol (a), high-density lipoprotein (HDL) cholesterol (b), total cholesterol (c), lauric acid (d), oleic acid (e), and linoleic acid (f). Three concentra-

with adjustment for sex, smoking status, ECOG-PS, PD-L1 expression, and line of treatment, LDL-cholesterol $(P$ $<0.001)$, HDL-cholesterol $(P=0.014)$, total cholesterol $(P=0.007)$, lauric acid $(P=0.015)$, myristic acid $(P=0.022)$, myristoleic acid $(P=0.035)$, stearic acid $(P=0.028)$, linoleic acid $(P=0.005)$, arachidic acid $(P=0.027)$, eicosadienoic acid $(P=0.017)$, dihomo- $\gamma$-linolenic acid $(P=0.036)$, and behenic acid levels $(P=0.032)$ were predictive of PFS (Table 2). In multiple-lipid analysis, four combinations of lipids were selected according to their correlations (Supplementary Methods). When LDL-cholesterol, HDL-cholesterol, lauric acid, myristoleic acid, stearic acid, eicosenoic acid, and dihomo- $\gamma$-linolenic acid were analyzed together, LDL-cholesterol $(P=0.001)$, HDL-cholesterol $(P=0.027)$, and lauric acid levels $(P=0.040)$ were predictive of PFS (Table 2). In the other three combinations, no lipid was predictive (Supplementary Table 5). tion categories were created for each lipid: low (less than the interquartile range [IQR], blue line), intermediate (within the IQR, green line), and high (higher than the IQR, red line)

\section{Associations between lipids and OS}

In the log-rank trend analyses of the Kaplan-Meier curves, OS increased with increasing LDL-cholesterol $(P<0.001$, Fig. 5a), HDL-cholesterol ( $P=0.029$, Fig. 5b), total cholesterol $(P=0.019$, Fig. 5c), lauric acid $(P=0.032$, Fig. 5 d $)$, oleic acid $(P=0.039$, Fig. 5e), linoleic acid $(P=0.001$, Fig. 5f), linolenic acid ( $P=0.017$, Fig. 6a), eicosadienoic acid $(P=0.011$, Fig. $6 \mathrm{~b})$, and lignoceric acid levels $(P=0.002$, Fig. 6c). Although not statistically significant, OS tended to increase with increasing myristic acid ( $P=0.111$, Fig. 6d), myristoleic acid $(P=0.098$, Fig. 6e), palmitoleic acid $(P=0.070$, Fig. 6f), stearic acid $(P=0.171$, Fig. 7a), eicosenoic acid $(P=0.158$, Fig. 7b), dihomo- $\gamma$ linolenic acid $(P=0.120$, Fig. $7 \mathrm{c})$, docosatetraenoic acid $(P=0.091$, Fig. $7 \mathrm{~d})$, and behenic acid levels $(P=0.058$, Fig. 7e). There was no association of OS with palmitic acid $(P=0.230$, Fig. 7 f $), \gamma$-linolenic acid $(P=0.488$, Fig. 8 a), 
a

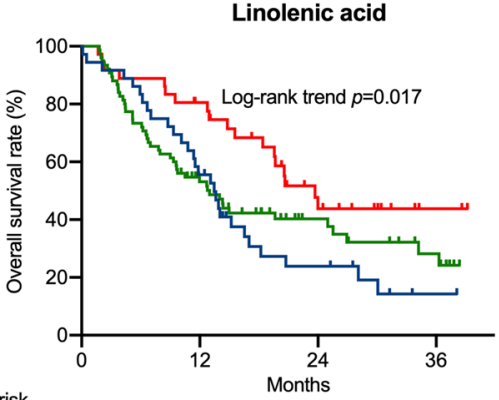

b

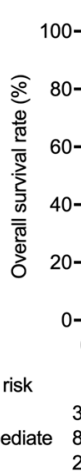

Eicosadienoic acid

og-rank trend $p=0.011$

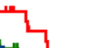

4.
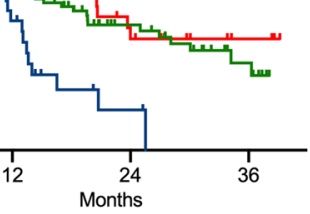

$\begin{array}{lllllll}\text { High } & 36 & 28 & 12 & 3 & \text { High } & 35 \\ \text { Intermediate } & 76 & 36 & 16 & 8 & \text { Intermediate } & 84\end{array}$

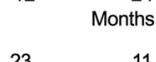

23
48 e

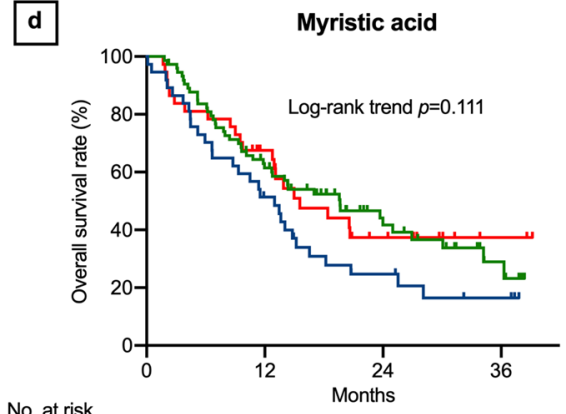

d

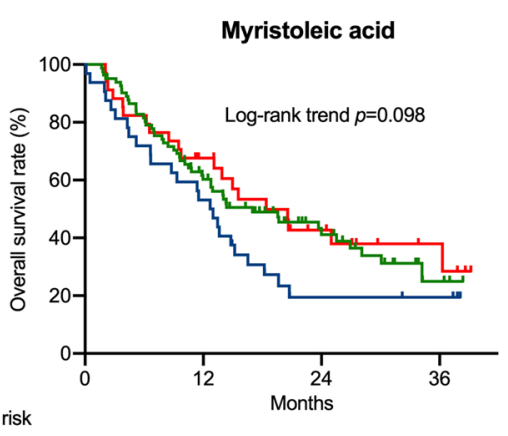

No. at risk

\section{High}

Intermediate 82

34
82
32

$\begin{array}{lcl}21 & 11 & 5 \\ 46 & 21 & 4 \\ 18 & 5 & 4\end{array}$
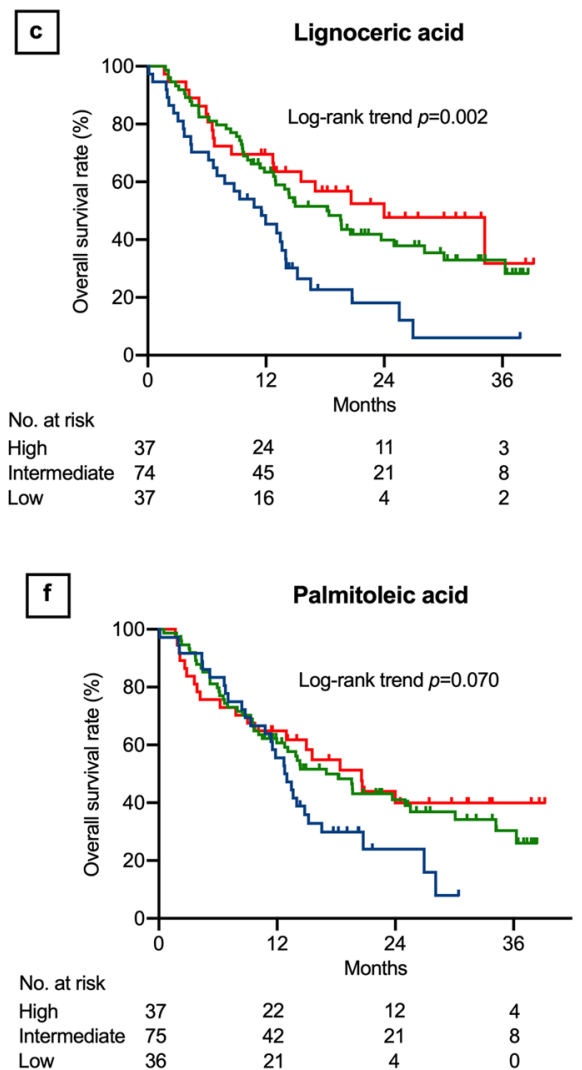

+ High Intermediate + Low

Fig. 6 Overall survival after nivolumab therapy according to serum lipid levels: part 2. Linolenic acid (a), eicosadienoic acid (b), lignoceric acid (c), myristic acid (d), myristoleic acid (e), and palmitoleic acid (f). Three concentration categories were created for each

arachidic acid $(P=0.341$, Fig. 8 b), eicosatrienoic acid $(P=0.241$, Fig. $8 \mathrm{c})$, arachidonic acid $(P=0.171$, Fig. $8 \mathrm{~d})$, eicosapentaenoic acid $(P=0.877$, Fig. 8e), docosapentaenoic acid $(P=0.220$, Fig. 8 f), docosahexaenoic acid $(P=0.348$, Supplementary Fig. 2a), and nervonic acid levels $(P=0.130$, Supplementary Fig. 2b).

In univariate Cox proportional hazard analyses, increased LDL-cholesterol $(P<0.001)$, HDL-cholesterol $(P=0.015)$, total cholesterol $(P=0.011)$, myristic acid $(P=0.025)$, myristoleic acid $(P=0.043)$, steric acid $(P=0.042)$, linoleic acid $(P=0.003)$, eicosadienoic acid $(P=0.016)$, behenic acid $(P=0.017)$, and lignoceric acid levels $(P=0.006)$ were predictive of OS, similarly as BMI, ECOG-PS, pathology, and PD-L1 expression (Supplementary Table 6). When each lipid was analyzed with adjustment for sex, BMI, ECOGPS, pathology, and PD-L1 expression, LDL-cholesterol $(P<0.001)$, HDL-cholesterol $(P=0.009)$, total cholesterol $(P=0.036)$, linoleic acid $(P=0.014)$, and lignoceric acid levels $(P=0.028)$ were significant predictive factors (Table 3). In multiple-lipid analysis, nine combinations of lipid: low (less than the interquartile range [IQR], blue line), intermediate (within the IQR, green line), and high (higher than the IQR, red line)

lipids were selected according to their correlations (Supplementary Methods). When LDL-cholesterol, HDLcholesterol, stearic acid, myristoleic acid, stearic acid, dihomo- $\gamma$-linolenic acid, and lignoceric acid were analyzed together, LDL-cholesterol $(P=0.005)$ and HDL-cholesterol $(P=0.035)$ were predictive of OS (Table 3$)$. In the other eight combinations, LDL-cholesterol and HDL-cholesterol were predictive of OS (Supplementary Table 7).

There was no significant association between lipid levels and ORR (Supplementary Table 8).

\section{Associations between lipid levels and the efficacy of cytotoxic chemotherapy}

One hundred and thirteen patients in the chemotherapy cohort had comparable demographics as nivolumab-treated patients (Supplementary Table 9).

In univariate Cox proportional hazard analysis, total cholesterol $(P=0.044)$, linoleic acid $(P=0.018)$, eicosadienoic 
a

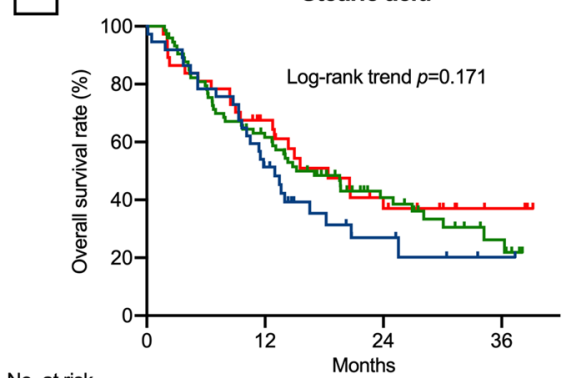

b

\section{at risk}

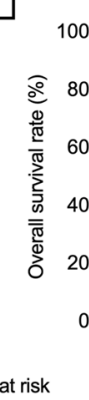

Eicosenoic acid

High
Intermediate
Low

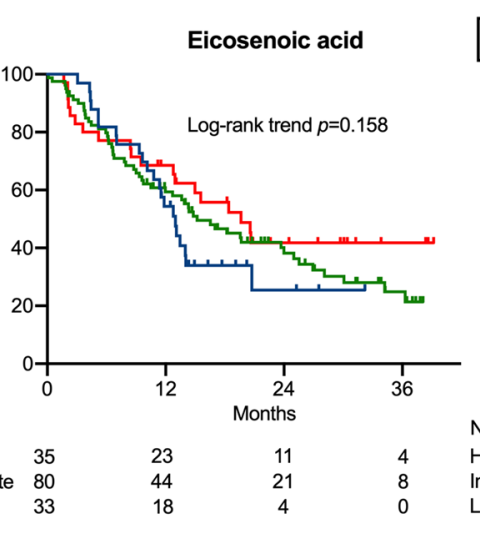

e
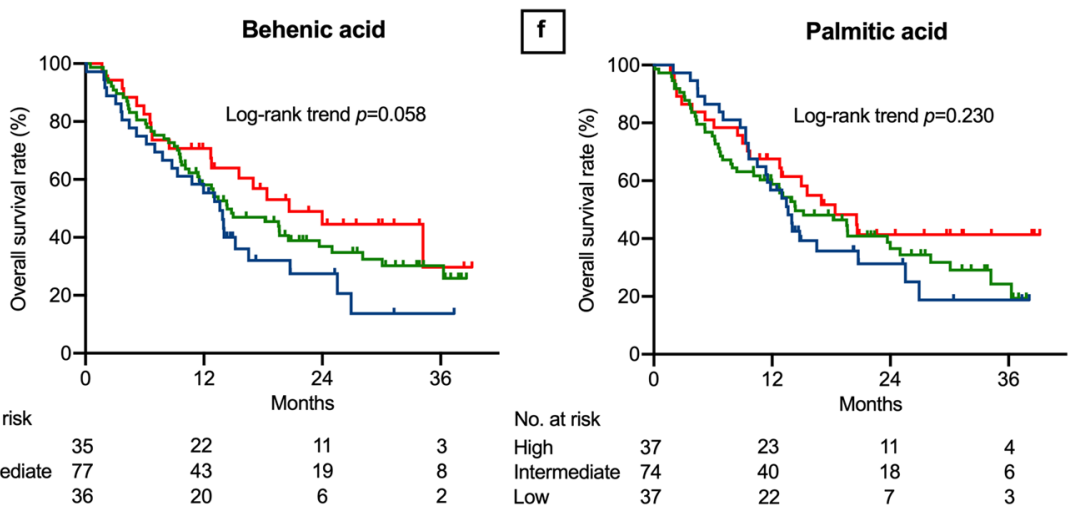

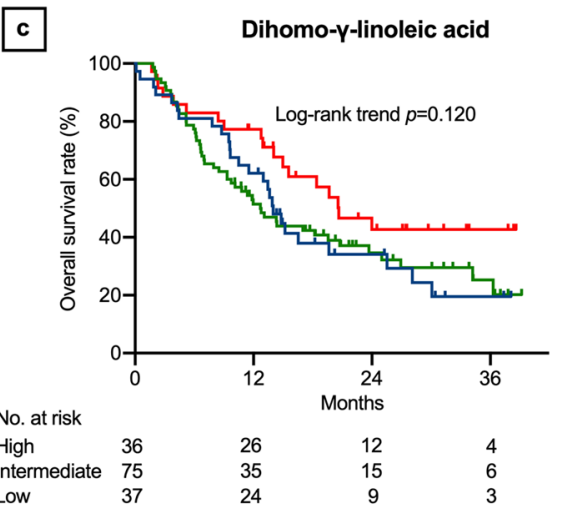

f
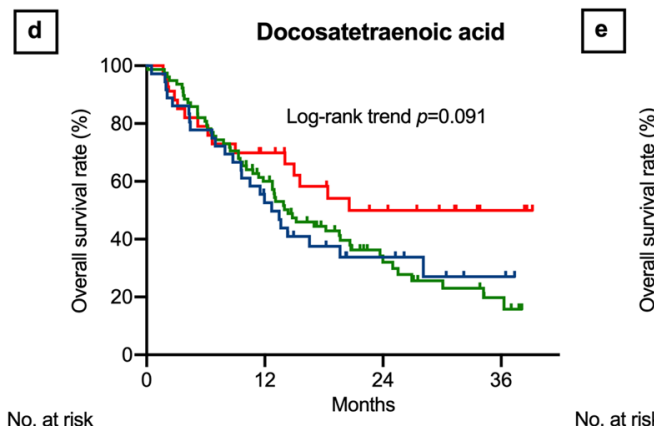

$\begin{array}{lllcl}\text { No. at risk } & & & & \\ \text { High } & 34 & 21 & 12 & 4 \\ \text { Intermediate } & 78 & 45 & 16 & 6 \\ \text { Low } & 36 & 19 & 8 & 3\end{array}$

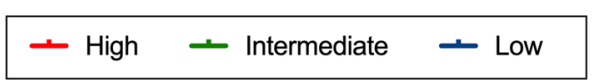

Fig. 7 Overall survival after nivolumab therapy according to serum lipid levels: part 3. Stearic acid (a), eicosenoic acid (b), dihomo- $\gamma$ linoleic acid (c), docosatetraenoic acid (d), behenic acid (e), and palmitic acid (f). Three concentration categories were created for each

acid $(P=0.037)$, arachidonic acid $(P=0.046)$, and docosatetraenoic acid levels $(P=0.010)$ were predictive of PFS, similarly as age, ECOG-PS, and the receipt of cisplatinbased therapy (vs. carboplatin-based therapy, Supplementary Table 10). After adjustment for age, sex, ECOG-PS, and the receipt of cisplatin-based therapy, no lipids were significantly predictive of PFS (Supplementary Table 10). Univariate Cox proportional hazard analysis identified no predictive factors for OS, and univariate logistic regression analysis indicated that no factors were predictive of ORR (Supplementary Tables 11-12).

\section{Discussion}

This was the first study to evaluate the association between multiple lipids and the efficacy of nivolumab in patients with previously treated NSCLC. Interestingly, we found that pretreatment serum cholesterol and long-chain fatty acid levels were significantly associated with PFS and OS, but lipid: low (less than the interquartile range [IQR], blue line), intermediate (within the IQR, green line), and high (higher than the IQR, red line)

not ORR, in patients treated with nivolumab, independent of ECOG-PS and PD-L1 expression. Conversely, no lipids were associated with the efficacy of cytotoxic chemotherapy. Serum lipid levels can be easily and noninvasively measured to assess patient status. In addition, lipids are natural products that can be administered easily and safely, and therefore, they have potential as therapeutic agents in combination with ICI therapy. Our data indicated the potential utility of lipids for predicting the efficacy of ICI therapy.

Increased serum lipid levels may compensate for lipid deprivation in immune cells in the tumor microenvironment. Cancer cells accumulate lipids in competition with immune cells, leading to in lipid deficiency in immune cells [7, 20-22]. It has been reported that increased exogenous lipids can potentially restore the activity of lipiddeficient immune cells in the tumor microenvironment. Mice with diet-induced hypercholesterolemia exhibited increased numbers of CD8+ and natural killer (NK) cells in hepatocellular carcinoma and adjacent tissues, leading to enhanced cytotoxic activity against cancer cells [30]. In 

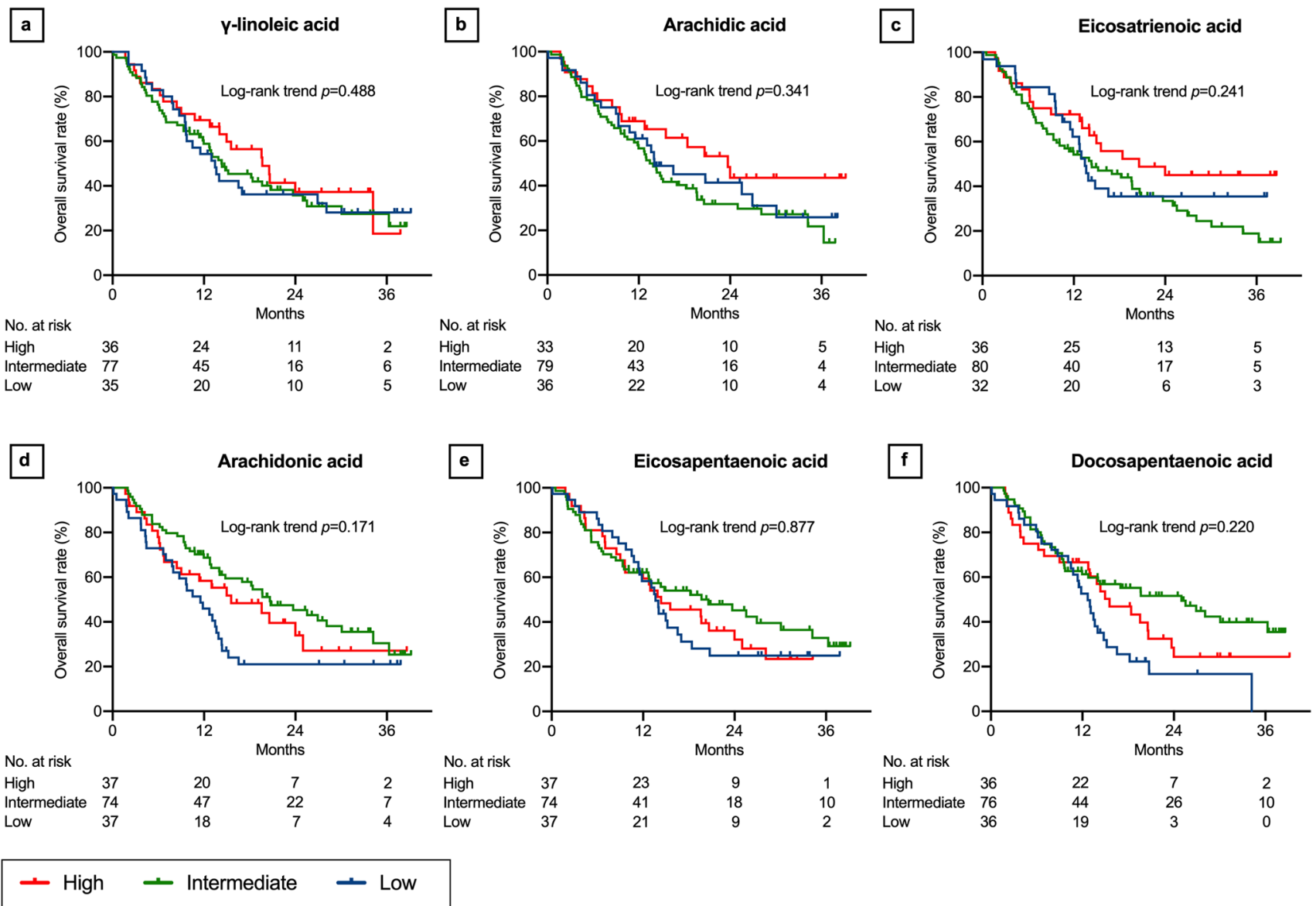

Fig. 8 Overall survival after nivolumab therapy according to serum lipid levels: part 4. $\gamma$-linoleic acid (a), arachidic acid (b), eicosatrienoic acid (c), arachidonic acid (d), eicosapentaenoic acid (e), and docosapentaenoic acid (f). Three concentration categories were cre-

patients with hepatocellular carcinoma, serum total cholesterol and LDL-cholesterol levels were positively correlated with the counts and cytotoxicity of intratumoral NK cells [30].

Another possible mechanism of the correlation between lipid levels and the efficacy of nivolumab was that cholesterol-induced $\mathrm{T}$ cell exhaustion can paradoxically increase the efficacy of anti-PD-1 therapy. It has been reported that increased extracellular cholesterol content induces immune checkpoint molecules on $\mathrm{T}$ cells including PD-1, leading to $\mathrm{T}$ cell exhaustion [31]. It is speculated that patients with cancer and elevated cholesterol levels exhibit PD-1-induced immune escape, increasing their susceptibility to anti-PD-1 therapy, whereas those without high cholesterol levels may experience immune escape caused by non-PD-1-induced pathways, leading to insusceptibility. Contrarily, cytotoxic chemotherapy does not confer any therapeutic advantage for PD-1-induced T cell exhaustion, and thus, this treatment did not influence the association between serum lipids and treatment efficacy. ated for each lipid: low (less than the interquartile range [IQR], blue line), intermediate (within the IQR, green line), and high (higher than the IQR, red line). Kaplan-Meier curves of docosahexaenoic acid and nervonic acid are presented in Supplementary Fig. 2

Similar paradoxical effects were also reported in obesity, an established risk factor for several cancers. In 331 patients who received immunotherapy for melanoma, obese patients (BMI $\geq 30 \mathrm{~kg} / \mathrm{m}^{2}$ ) exhibited significantly longer PFS and OS than those with normal BMI $\left(18.5-24.9 \mathrm{~kg} / \mathrm{m}^{2}\right)$, but this was not replicated in patients who received chemotherapy [32]. Similar results were reported in 250 patients with a variety of cancers who received immunotherapies [33]. Mice with diet-induced obesity displayed increased PD-1-positive T cell exhaustion in the tumor microenvironment and greater tumor growth control diet-fed mice. Inversely, the efficacy of anti-PD-1 treatment was better in obese mice than in controls [33]. The study focused on increased leptin levels attributable to obesity as the cause of $\mathrm{T}$ cell exhaustion; however, the precise mechanisms underlying the improved efficacy of anti-PD-1 treatment in obesity were not clarified. The current study did not include any patients with BMI $\geq 30 \mathrm{~kg} / \mathrm{m}^{2}$, and BMI was not associated with the levels of any lipids or the efficacy of nivolumab. However, 
Table 3 Multivariate cox proportional hazard analyses of overall survival

\begin{tabular}{|c|c|c|c|c|}
\hline \multirow[b]{2}{*}{ Variables } & \multicolumn{2}{|c|}{ Single-lipid analysis } & \multicolumn{2}{|c|}{ Multiple-lipid analysis } \\
\hline & Hazard ratio & $P$ & Hazard ratio & $P$ \\
\hline LDL-cholesterol $^{\mathrm{a}}$ & $0.87(0.80-0.94)$ & $<0.001$ & $0.86(0.77-0.96)$ & 0.005 \\
\hline HDL-cholesterol $^{\mathrm{a}}$ & $0.76(0.62-0.93)$ & 0.009 & $0.81(0.66-0.99)$ & 0.035 \\
\hline Total cholesterol $^{\mathrm{a}}$ & $0.95(0.90-0.99)$ & 0.036 & & \\
\hline Lauric acid ${ }^{\mathrm{b}}$ & $0.91(0.68-1.07)$ & 0.375 & & \\
\hline Myristic acid ${ }^{\mathrm{b}}$ & $0.93(0.82-1.04)$ & 0.251 & & \\
\hline Myristoleic acid & $0.92(0.79-1.05)$ & 0.289 & $0.91(0.75-1.11)$ & 0.346 \\
\hline Palmitic acid ${ }^{\mathrm{d}}$ & $0.98(0.89-1.08)$ & 0.678 & & \\
\hline Palmitoleic acid ${ }^{\mathrm{b}}$ & $1.00(0.94-1.05)$ & 0.916 & & \\
\hline Stearic acid ${ }^{\mathrm{d}}$ & $0.85(0.58-1.23)$ & 0.405 & $1.65(0.90-3.01)$ & 0.112 \\
\hline Oleic acid ${ }^{\mathrm{d}}$ & $1.00(0.92-1.09)$ & 0.915 & & \\
\hline Linoleic acid ${ }^{\mathrm{d}}$ & $0.87(0.79-0.97)$ & 0.014 & & \\
\hline$\gamma$-linolenic acid ${ }^{c}$ & $1.00(0.96-1.03)$ & 0.829 & & \\
\hline Linolenic acid ${ }^{\mathrm{c}}$ & $0.99(0.98-1.01)$ & 0.554 & & \\
\hline Arachidic acid ${ }^{c}$ & $0.97(0.88-1.06)$ & 0.516 & & \\
\hline Eicosenoic acid ${ }^{c}$ & $0.99(0.89-1.08)$ & 0.783 & & \\
\hline Eicosadienoic acid ${ }^{c}$ & $0.92(0.83-1.01)$ & 0.105 & & \\
\hline Eicosatrienoic acid ${ }^{c}$ & $0.98(0.88-1.09)$ & 0.759 & & \\
\hline Dihomo- $\gamma$-linolenic acid & $0.99(0.98-1.01)$ & 0.441 & $1.00(0.98-1.02)$ & 0.831 \\
\hline Arachidonic acid ${ }^{\mathrm{b}}$ & $0.97(0.93-1.02)$ & 0.296 & & \\
\hline Eicosapentaenoic acid ${ }^{b}$ & $1.02(0.95-1.08)$ & 0.513 & & \\
\hline Behenic acid $^{c}$ & $0.96(0.91-1.00)$ & 0.086 & & \\
\hline Docosatetraenoic acid & $0.96(0.85-1.07)$ & 0.479 & & \\
\hline Docosapentaenoic acid & $1.00(0.97-1.03)$ & 0.810 & & \\
\hline Lignoceric acid ${ }^{\mathrm{c}}$ & $0.95(0.90-0.99)$ & 0.028 & $0.99(0.93-1.06)$ & 0.808 \\
\hline Docosahexaenoic acid ${ }^{b}$ & $0.98(0.92-1.03)$ & 0.400 & & \\
\hline Nervonic acid $^{\mathrm{b}}$ & $1.06(0.85-1.33)$ & 0.581 & & \\
\hline
\end{tabular}

The hazard ratio was adjusted for sex, body mass index, Eastern Cooperative Oncology Group performance status, pathology, and programmed death ligand-1 expression. Total cholesterol, myristic acid, linoleic acid, and lignoceric acid were excluded from the multiple-lipid analysis because of their strong correlations with other lipids, and the results of the combination of the excluded lipids are presented in Supplementary Table 7

${ }^{a}$ per $10 \mathrm{mg} / \mathrm{dL}$ increase

${ }^{\mathrm{b}}$ per $10 \mathrm{mg} / \mathrm{dL}$ increase

${ }^{c}$ per $1 \mathrm{mg} / \mathrm{dL}$ increase

${ }^{\mathrm{d}}$ per $100 \mathrm{mg} / \mathrm{dL}$ increase obesity is often associated with hyperlipidemia, which can affect obesity-associated immune alterations.

Interestingly, $\omega-6$ PUFAs (linoleic acid, arachidic acid, eicosadienoic acid, and dihomo- $\gamma$-linolenic acid) and saturated fatty acids (lauric acid, myristic acid, stearic acid, behenic acid, and lignoceric acid), which are known to promote pro-inflammatory response, displayed positive associations with treatment efficacy. On the contrary, $\omega-3$ PUFAs (linolenic acid, eicosatetraenoic acid, eicosapentaenoic acid, docosapentaenoic acid, or docosahexaenoic acid), which are known to promote anti-inflammatory responses, exhibited no association with efficacy. Because of their counter-regulatory roles in immunity, $\omega-3$ PUFAs are considered beneficial for inflammatory diseases, cardiovascular diseases, and cancers, whereas $\omega-6$ PUFAs and saturated fatty acids are considered risk factors $[17,18,34]$. However, in the context of immune therapy, pro-inflammatory responses have potential benefits.

However, controversies exist regarding the roles of cholesterol and fatty acids in cancer immunity. Lipid cannot always provide favorable conditions for cancer immunity. It is reported that LDL-cholesterol inhibited $\mathrm{T}$ cell activation, resulting in impaired antitumor function [35]. Increased cholesterol and/or fatty acid levels have the potential to attenuate anticancer immunity via activation suppressor immune cells, such as regulatory $\mathrm{T}$ cells (Tregs), myeloid-derived 
suppressor cells (MDSCs), and immunosuppressive tumorassociated macrophages (TAMs) [20, 22, 24]. The difference in cancer immunity between LDL- and HDL-cholesterol is also unclear. Generally, LDL and HDL exert counterregulatory effects on cholesterol transport: LDL promotes inflammation via cholesterol influx into immune cells, whereas HDL reduces inflammation via cholesterol efflux [8]. If cholesterol-induced pro-inflammatory responses are beneficial for anticancer immunity, then increased levels of HDL-cholesterol would be unfavorable. However, in the current study, increased HDL-cholesterol levels were associated with longer PFS and OS independent of LDLcholesterol levels. The tumor microenvironment has complicated cross-interactions among tumor cells, effector cells, and regulatory immune cells, and metabolism is uniquely regulated in each cell type. Further studies are warranted to understand the complex mechanism of lipid metabolism in cancer immunity.

The current study had three main limitations. First, it is unknown which lipids have actual and/or the most relevant immunomodulatory activities that affect the efficacy of nivolumab. The multiple-lipid analysis using the selected combinations identified certain lipids as major contributing factors. However, selection based on correlation coefficients could not completely eliminate potential confounding. This is because lipids have a complicated metabolic cascade in which they are directly and/or indirectly associated with each other, and lipid composition varies among foods (major source of lipids for humans). In addition, it is also possible that selection based on correlations ignored the potential importance of the excluded lipids. Second, the differences in lipid composition and/or concentration between peripheral blood and the tumor microenvironment are unknown. In fact, cancer tissue is reported to have twofold higher free cholesterol and 3.5-fold higher esterified cholesterol levels, probably because of increased intake by tumor cells [36]. Third, serum lipids levels were provisionally determined according to the statistical distribution of the limited number of study patients. It is unknown whether higher serum levels will always lead to better clinical outcomes. In addition, it is also unknown whether increased lipid levels following supplemental treatment have beneficial effects. The regulation of lipid metabolism can potentially enhance the efficacy of immunotherapy, and this strategy has attracted attention as a novel therapeutic option [37-39]. Further studies are needed to evaluate the optimal levels and therapeutic benefits of lipids in ICI therapy.

In conclusion, increased serum cholesterol and long-chain fatty acid levels were associated with better PFS and OS in patients with previously treated NSCLC who received nivolumab. Serum lipids may be useful for predicting the efficacy of ICIs.
Supplementary Information The online version contains supplementary material available at https://doi.org/10.1007/s00262-021-02979-4.

Acknowledgements We would like to acknowledge patients and their families. We thank Joe Barber Jr., PhD, from Edanz Group (https:// en-author-services.edanz.com/ac) for editing a draft of this manuscript.

Authors' contribution MK has full access to all of the data in the study and takes responsibility for the integrity of the data and the accuracy of the data analysis. MK participated in concept and design, acquisition, analysis, and interpretation of data, statistical analyses, and drafting of the manuscript. NI participated in concept and design, interpretation of data, drafting of the manuscript, and supervision. YI and KY participated in concept and design, acquisition, analysis, and interpretation of data, statistical analyses, technical supports, and drafting of the manuscript. KM participated in concept and design, interpretation of data, statistical analyses. HH, YS, KF, TF, NE, and YN participated in concept and design, interpretation of data, and drafting of the manuscript. KA, TU, MF, TM, SM, DH, MT, HK, HM, NI, YK, MN, and YI participated in concept and design, data acquisition, and drafting of the manuscript. TS participated in concept and design, drafting of the manuscript, and supervision.

Funding This research was supported by the Japan Society for the Promotion of Science [19K17631].

Data availability The datasets used and/or analyzed during the current study are available from the corresponding author on reasonable request.

\section{Declarations}

Conflict of interest All authors have no conflict of interest in relation to this manuscript.

Consent to participate Each patient provided written informed consent.

Ethical approval The study protocol was approved by the Institutional Review Board of Hamamatsu University School of Medicine (No. 19-083).

Open Access This article is licensed under a Creative Commons Attribution 4.0 International License, which permits use, sharing, adaptation, distribution and reproduction in any medium or format, as long as you give appropriate credit to the original author(s) and the source, provide a link to the Creative Commons licence, and indicate if changes were made. The images or other third party material in this article are included in the article's Creative Commons licence, unless indicated otherwise in a credit line to the material. If material is not included in the article's Creative Commons licence and your intended use is not permitted by statutory regulation or exceeds the permitted use, you will need to obtain permission directly from the copyright holder. To view a copy of this licence, visit http://creativecommons.org/licenses/by/4.0/.

\section{References}

1. Borghaei H, Paz-Ares L, Horn L, Spigel DR, Steins M, Ready NE et al (2015) Nivolumab versus docetaxel in advanced nonsquamous non-small-cell lung cancer. N Engl J Med 373(17):1627-1639 
2. Brahmer J, Reckamp KL, Baas P, Crinò L, Eberhardt WEE, Poddubskaya E et al (2015) Nivolumab versus docetaxel in advanced squamous-cell non-small-cell lung cancer. N Engl J Med 373(2):123-135

3. Gibney GT, Weiner LM, Atkins MB (2016) Predictive biomarkers for checkpoint inhibitor-based immunotherapy. Lancet Oncol 17(12):e542-e551

4. Sharma P, Allison JP (2015) The future of immune checkpoint therapy. Science 348(6230):56-61

5. Pearce EL, Pearce EJ (2013) Metabolic pathways in immune cell activation and quiescence. Immunity 38(4):633-643

6. Fox CJ, Hammerman PS, Thompson CB (2005) Fuel feeds function: energy metabolism and the T-cell response. Nat Rev Immunol 5(11):844-852

7. Kishton RJ, Sukumar M, Restifo NP (2017) Metabolic regulation of T cell longevity and function in tumor immunotherapy. Cell Metab 26(1):94-109

8. Tall Alan R, Yvan-Chrvet L (2015) Cholesterol, inflammation and innate immunity. Nat Rev Immunol 15(2):104-16

9. O'Brien KL, Finlay DK (2019) Immunometabolism and natural killer cell responses. Nat Rev Immunol 19(5):282-290

10. Newton R, Priyadharshini B, Turka LA (2016) Immunometabolism of regulatory T cells. Nat Immunol 17(6):618-625

11. Lochner M, Berod L, Sparwasser T (2015) Fatty acid metabolism in the regulation of $\mathrm{T}$ cell function. Trends Immunol 36(2):81-91

12. Moon YW, Hajjar J, Hwu P, Naing A (2015) Targeting the indoleamine 2,3-dioxygenase pathway in cancer. J Immunother Cancer 3(1):1-10

13. Munn DH, Mellor AL (2016) IDO in the tumor microenvironment: inflammation, counter-regulation and tolerance. Trends Immnol 37(3):193-207

14. Karayama M, Masuda J, Mori K, Yasui H, Hozumi H, Suzuki $Y$ et al (2020) Comprehensive assessment of multiple tryptophan metabolites as potential biomarkers for immune checkpoint inhibitors in patients with non-small cell lung cancer. Clin Transl Oncol. https://doi.org/10.1007/s12094-020-02421-8

15. Chyu KY, Lio WM, Dimayuga PC, Zhou J, Zhao X, Yano J et al (2014) Cholesterol lowering modulates $T$ cell function in vivo and in vitro. PLoS One 9(3):e92095

16. Surls J, Nazarov-Stoica C, Kehl M, Olsen C, Casares S, Brumeanu TD (2012) Increased membrane cholesterol in lymphocytes diverts T-cells toward an inflammatory response. PLoS ONE 7(6):1-13

17. Haghikia A, Jörg S, Duscha A, Berg J, Manzel A, Waschbisch A et al (2015) Dietary fatty acids directly impact central nervous system autoimmunity via the small intestine. Immunity 43(4):817-829

18. Hammer A, Schliep A, Jörg S, Haghikia A, Gold R, Kleinewietfeld $M$ et al (2017) Impact of combined sodium chloride and saturated long-chain fatty acid challenge on the differentiation of $\mathrm{T}$ helper cells in neuroinflammation. J Neuroinflammation 14(1):1-9

19. Weatherill AR, Lee JY, Zhao L, Lemay DG, Youn HS, Hwang DH (2005) Saturated and polyunsaturated fatty acids reciprocally modulate dendritic cell functions mediated through TLR4. J Immunol 174(9):5390-5397

20. Wang X, Ping FF, Bakht S, Ling J, Hassan W (2019) Immunometabolism features of metabolic deregulation and cancer. J Cell Mol Med 23(2):694-701

21. Mancini R, Noto A, Pisanu ME, De Vitis C, Maugeri-Saccà M, Ciliberto G (2018) Metabolic features of cancer stem cells: the emerging role of lipid metabolism. Oncogene 37(18):2367-2378

22. Dyck L, Lynch L (2018) Cancer, obesity and immunometabolismconnecting the dots. Cancer Lett 417:11-20
23. Mok EHK, Lee TKW (2020) The pivotal role of the dysregulation of cholesterol homeostasis in cancer: implications for therapeutic targets. Cancers (Basel) 12(6):1410

24. Gil-De-gómez L, Balgoma D, Montero O (2020) Lipidomic-based advances in diagnosis and modulation of immune response to cancer. Metabolites 10(8):1-15

25. Gutiérrez S, Svahn SL, Johansson ME (2019) Effects of omega-3 fatty acids on immune cells. Int J Mol Sci 20(20):5028

26. Mukaro VR, Costabile M, Murphy KJ, Hii CS, Howe PR, Ferrante A (2008) Leukocyte numbers and function in subjects eating n-3 enriched foods: selective depression of natural killer cell levels. Arthritis Res Ther 10(3):1-11

27. Wanten GJA, Janssen FP, Naber AHJ (2002) Saturated triglycerides and fatty acids activate neutrophils depending on carbon chain-length. Eur J Clin Invest 32(4):285-289

28. Inoue $\mathrm{Y}$, Yoshimura $\mathrm{K}$, Nishimoto $\mathrm{K}$, Inui $\mathrm{N}$, Karayama $\mathrm{M}$, Yasui $\mathrm{H}$ et al (2020) Evaluation of programmed death ligand 1 (PD-L1) gene amplification and response to nivolumab monotherapy in non-small cell lung cancer. JAMA Netw Open 3(9):e2011818

29. von Elm E, Altman DG, Egger M, Pocock SJ, Gøtzsche PC, Vandenbroucke JP (2014) The strengthening the reporting of observational studies in epidemiology (STROBE) statement: guidelines for reporting observational studies. Int J Surg 12(12):1495-1499

30. Qin W, Yang Z, Li M, Chen Y, Zhao X, Qin Y et al (2020) High serum levels of cholesterol increase antitumor functions of nature killer cells and reduce growth of liver tumors in mice. Gastroenterology 158(6):1713-1727

31. Ma X, Bi E, Lu Y, Su P, Huang C, Liu L et al (2019) Cholesterol induces $\mathrm{CD} 8+\mathrm{T}$ cell exhaustion in the tumor microenvironment. Cell Metab 30(1):143-156.e5

32. McQuade JL, Daniel CR, Hess KR, Mak C, Wang DY, Rai RR et al (2018) Association of body-mass index and outcomes in patients with metastatic melanoma treated with targeted therapy, immunotherapy, or chemotherapy: a retrospective, multicohort analysis. Lancet Oncol 19(3):310-322

33. Wang Z, Aguilar EG, Luna JI, Dunai C, Khuat LT, Le CT et al (2019) Paradoxical effects of obesity on T cell function during tumor progression and PD-1 checkpoint blockade. Nat Med 25(1):141-151

34. Saini RK, Keum YS (2018) Omega-3 and omega-6 polyunsaturated fatty acids: dietary sources, metabolism, and significance-a review. Life Sci 2018(203):255-267

35. Rodrigues NV, Correia DV, Mensurado S, Nobrega-Pereira S, deBarros A, Kyle-Cezar F et al (2018) Low-density lipoprotein uptake inhibits the activation and antitumor functions of human Vg9Vd2 T cells. Cancer Immunol Res 6(4):448-457

36. Dessi S, Batetta B, Pulisci D, Spano O, Cherchi R, Lanfranco $\mathrm{G}$ et al (1992) Altered pattern of lipid metabolism in patients with lung cancer. Oncology 49(6):436-441

37. Chowdhury PS, Chamoto K, Kumar A, Honjo T (2018) PPARinduced fatty acid oxidation in $\mathrm{T}$ cells increases the number of tumor-reactive CD8 + T cells and facilitates anti-PD-1 therapy. Cancer Immunol Res 6(11):1375-1387

38. Chamoto K, Chowdhury PS, Kumar A, Sonomura K, Matsuda F, Fagarasan S et al (2017) Mitochondrial activation chemicals synergize with surface receptor PD-1 blockade for T cell-dependent antitumor activity. Proc Natl Acad Sci U S A 114(5):E761-E770

39. Saibil SD, St Paul M, Laister RC, Garcia-Batres CR, IsraniWinger K, Elford AR et al (2019) Activation of peroxisome proliferator-activated receptors a and D synergizes with inflammatory signals to enhance adoptive cell therapy. Cancer Res 79(3):445-51 
Publisher's Note Springer Nature remains neutral with regard to jurisdictional claims in published maps and institutional affiliations.

\section{Authors and Affiliations}

\section{Masato Karayama ${ }^{1,2}$ (D) Naoki Inui ${ }^{3} \cdot$ Yusuke Inoue $^{2} \cdot$ Katsuhiro Yoshimura $^{2} \cdot$ Kazutaka Mori $^{4} \cdot$ Hironao Hozumi $^{2} \cdot$ Yuzo Suzuki $^{2} \cdot$ Kazuki Furuhashi $^{2} \cdot$ Tomoyuki Fujisawa $^{2} \cdot$ Noriyuki Enomoto $^{2} \cdot$ Yutaro Nakamura $^{2} \cdot$ Kazuhiro Asada $^{5}$. Tomohiro Uto $^{6}$. Masato Fujii ${ }^{7}$. Takashi Matsui ${ }^{8}$. Shun Matsuura ${ }^{9}$. Dai Hashimoto ${ }^{10}$ - Mikio Toyoshima ${ }^{11}$. Hideki Kusagaya $^{12} \cdot$ Hiroyuki Matsuda ${ }^{13} \cdot$ Nao Inami $^{4} \cdot$ Yusuke Kaida $^{14} \cdot$ Mitsuru Niwa $^{15} \cdot$ Yasuhiro Ito $^{16}$. Takafumi Suda²}

1 Department of Chemotherapy, Hamamatsu University School of Medicine, 1-20-1 Handayama, Hamamatsu 431-3192, Japan

2 Second Division, Department of Internal Medicine, Hamamatsu University School of Medicine, 1-20-1 Handayama, Hamamatsu 431-3192, Japan

3 Department of Clinical Pharmacology and Therapeutics, Hamamatsu University School of Medicine, 1-20-1 Handayama, Hamamatsu 431-3192, Japan

4 Department of Respiratory Medicine, Shizuoka City Shimizu Hospital, 1231 Miyakami, Shizuoka 424-8636, Japan

5 Department of Respiratory Medicine, Shizuoka General Hospital, 4-27-1 Kita-ando, Shizuoka 420-0881, Japan

6 Department of Respiratory Medicine, Iwata City Hospital, 513-2 Ohkubo, Iwata 438-8550, Japan

7 Department of Respiratory Medicine, Shizuoka City Hospital, 10-93 Ote-cho, Shizuoka 420-8630, Japan

8 Department of Respiratory Medicine, Seirei Mikatahara General Hospital, 3453 Mikatahara-cho, Hamamatsu 433-8558, Japan
9 Department of Respiratory Medicine, Fujieda Municipal General Hospital, 4-1-11 Surugadai, Fujieda 426-8677, Japan

10 Department of Respiratory Medicine, Seirei Hamamatsu General Hospital, 2-12-12 Sumiyoshi, Hamamatsu 430-8558, Japan

11 Department of Respiratory Medicine, Hamamatsu Rosai Hospital, 25 Shougen-cho, Hamamatsu 430-8525, Japan

12 Department of Respiratory Medicine, Shizuoka Saiseikai Hospital, 1-1-1 Oshika, Shizuoka 422-8527, Japan

13 Department of Respiratory Medicine, Japanese Red Cross Shizuoka Hospital, 8-2 Otemachi, Shizuoka 420-0853, Japan

14 Department of Respiratory Medicine, Ensyu Hospital, 1-1-1 Chuou, Hamamatsu 430-0929, Japan

15 Department of Respiratory Medicine, Hamamatsu Medical Center, 328 Tomitsuka-cho, Hamamatsu 432-8580, Japan

16 Department of Respiratory Medicine, National Hospital Organization Tenryu Hospital, 4201-2 Oro, Hamamatsu 434-8511, Japan 